\title{
Urogenital infections
}

\section{Florian Wagenlehner ${ }^{1}$}

Published online: 2 December 2019

c) Springer-Verlag GmbH Germany, part of Springer Nature 2019

Urological infections are increasingly posing threats to our patients, both in the outpatient and in the hospitalized setting. We have to consider that infections always are potentially communicable, and so is antimicrobial resistance. This makes infections in urology special and different from all other urological diseases. An antibiotic that is given to a patient with a urinary catheter and asymptomatic bacteriuria, which is not indicated, may induce emergence of resistance in this patient's microbiome. The antibiotic might not necessarily have a negative impact on this patient, but the resistant bacterium could be transmitted to an immunosuppressed patient who has undergone a renal transplant, and induce a life threatening infection. Therefore, urologists always have a responsibility towards the patient as well as the environment, when managing infections.

This topic issue reflects the wide scope of infections in urology. It summarizes the evidence from prevention, to diagnosing and evaluating infections, to treating infections in different settings. Medina-Polo et al. [1] present a cohort study, where the effect of protocols, aiming to reduce healthcare associated infections, were studied over several years. In this study, they could show that implementation of protocols for diagnosis and empirical treatment of health-care associated infections in urological wards dramatically reduced the rate of urological infections. Bruyere et al. [2] present a systematic literature survey on non-antibiotic measures for prophylaxis of postoperative infections. They show that apart from antibiotic prophylaxis various other measures to reduce postoperative infections in urology should be known and considered by anesthesiologists and surgeons. A very specific problem for urologists is infectious complications after transrectal prostate biopsy. Bjerklund Johansen et al. [3] start with a case report of a patient dying after transrectal prostate biopsy and discuss several important issues such as

Florian Wagenlehner

Florian.Wagenlehner@chiru.med.uni-giessen.de

1 Clinic of Urology, Pediatric Urology and Andrology, Justus Liebig University Giessen, Giessen, Germany risk factors and increasing antimicrobial resistance, reflected in the Norwegian patient registry for prostate biopsies from 2011 to 2017. The article by Tandogdu et al. [4] shows how mathematical modelling by Bayesian statistics can help improve empirical antibiotic treatment based on a conditionspecific surveillance of antimicrobial resistance, rather than descriptive surveillance of pathogen resistance only.

Novel diagnostic techniques such as metagenomics are discussed by Dixon et al. [5]. Their paper explores the limitations of traditional methods of culture and sensitivity and discusses recent studies involving new high-throughput genomic technologies in urological basic and clinical research, demonstrating the advances made in the urinary microbiome in its entire spectrum of pathogens and the first attempts of clinical implementation in several areas of urology. Batura and Gopal Rao [6] performed a systematic literature search and reviewed the evidence on the utility and role of nephrostomy urine cultures. They showed very nicely that nephrostomy urine cultures provide benefit in clinical practice and can help support treatment decisions and antimicrobial stewardship. They are particularly useful in selecting appropriate antimicrobial treatment of urosepsis following upper urinary tract obstruction, but do not appear to be of advantage for routine nephrostomy exchange.

In the paper by Laan et al. [7] patient's knowledge and experience with catheters were assessed by means of a selfreported questionnaire. They showed that many patients lack knowledge about the indication for having a urinary or a peripheral intravenous catheter. They conclude that there is substantial room for quality improvement initiatives, for stimulating patients to actively participate in their treatment and asking for removal of catheters. Alidjanov et al. [8] present the Acute Cystitis Symptom Score (ACSS) and its relevance in the light of the updated EMA and FDA guidelines for clinical diagnosis of uncomplicated cystitis. They conclude that the ACSS can be recommended for epidemiological and interventional studies, and allows women to establish self-diagnosis of acute uncomplicated cystitis.

Mangir et al. [9] discuss pathophysiological issues in complications related to vaginal mesh implants. They 
conclude that there is evidence that vaginal meshes are associated with an unfavourable host response at the site of implantation and suggest more basic research to be performed to identify the biological mechanisms causing a sustained inflammation in patients suffering from mesh complications. Ness and Olsburgh [10] write about urinary tract infections in kidney transplant patients. They emphasize that understanding and management of UTI in transplant patients require appreciation of the unique anatomy and physiology in this specific situation. Antimicrobial resistance is especially important in this population of immunosuppressed patients. Lastly, Kulchavenya et al. [11] discuss the challenges in urogenital tuberculosis and present their results of the analysis of 142 patients suffering from urogenital tuberculosis. It becomes obvious that the diagnosis and identification of $M$. tuberculosis are difficult in these patients. Therefore, the most advanced microbiological technology should be used to establish the correct diagnosis and multiple diagnostic approaches should be employed.

This topic issue of the World Journal of Urology on urological infections provides a large array of aspects of urological infections written by authors from all around the world. It shows that urologists worldwide are faced by the same problems, and are asking for new approaches to improve prevention and treatment of infections in urology.

Vladimir Mouraviev, who is the co-author of two papers in this topic issue [3,5], died of acute illness during the preparation of this topic issue. He was a highly valued member of the European Section of Infection in Urology of EAU, a dedicated scientist, investigating most modern technology, such as next generation sequencing in microbiological diagnosis, and a good friend to all of the authors. Our thoughts are with his wife Tatiana and their two daughters.

\section{References}

1. Medina-Polo J, Gil-Moradillo J, Justo-Quintas J, GonzálezPadilla DA, García-Rojo E, González-Díaz A, Abad-López P, Hernández-Arroyo M, Santos-Pérez de la Blanca R, Peña-Vallejo
H, Téigell-Tobar J, López-Medrano F, Tejido-Sánchez Á (2019) Prevention of healthcare-associated infections (HAIs) in a surgical urology ward: observational study-analysis of the problem and strategies for implementation. World J Urol. https://doi. org/10.1007/s00345-019-02648-3

2. Bruyere F, Pilatz A, Boehm A, Pradere B, Wagenlehner F, Vallee M (2019) Associated measures to antibiotic prophylaxis in urology. World J Urol. https://doi.org/10.1007/s00345-019-02854-z

3. Johansen TEB, Zahl PH, Baco E, Bartoletti R, Bonkat G, Bruyere F, Cai T, Cek M, Kulchavenya E, Köves B, Mouraviev V, Pilatz A, Tandogdu Z, Tenke P, Wagenlehner FME (2019) Antibiotic resistance, hospitalizations, and mortality related to prostate biopsy: first report from the Norwegian Patient Registry. World J Urol. https://doi.org/10.1007/s00345-019-02837-0

4. Tandogdu Z, Koves B, Cai T, Cek M, Tenke P, Naber K, Wagenlehner F, Bjerklund Johansen TE (2019) Condition-specifc surveillance in health care-associated urinary tract infections as a strategy to improve empirical antibiotic treatment: an epidemiological modelling study. World J Urol. https://doi.org/10.1007/ s00345-019-02963-9

5. Dixon M, Stefil M, McDonald M, Bjerklund-Johansen TE, Naber K, Wagenlehner F, Mouraviev V (2019) Metagenomics in diagnosis and improved targeted treatment of UTI. World J Urol. https:// doi.org/10.1007/s00345-019-02731-9

6. Batura D, Gopal Rao G (2019) A systematic review of the clinical significance of nephrostomy urine cultures. World J Urol. https:// doi.org/10.1007/s00345-019-02663-4

7. Laan BJ, Nieuwkerk PT, Geerlings SE (2019) Patients knowledge and experience with urinary and peripheral intravenous catheters. World J Urol. https://doi.org/10.1007/s00345-018-02623-4

8. Alidjanov JF, Naber KG, Pilatz A, Radzhabov A, Zamuddinov M, Magyar A, Tenke P, Wagenlehner FM (2019) Evaluation of the draft guidelines proposed by EMA and FDA for the clinical diagnosis of acute uncomplicated cystitis in women. World J Urol. https://doi.org/10.1007/s00345-019-02761-3

9. Mangir N, Roman S, Chapple CR, MacNeil S (2019) Complications related to use of mesh implants in surgical treatment of stress urinary incontinence and pelvic organ prolapse: infection or inflammation? World J Urol. https://doi.org/10.1007/s00345-01902679-w

10. Ness D, Olsburgh J (2019) UTI in kidney transplant. World J Urol. https://doi.org/10.1007/s00345-019-02742-6

11. Kulchavenya E, Kholtobin D, Shevchenko S (2019) Challenges in urogenital tuberculosis. World J Urol. https://doi.org/10.1007/ s00345-019-02767-x

Publisher's Note Springer Nature remains neutral with regard to jurisdictional claims in published maps and institutional affiliations. 